\title{
对二茂铁苯甲酰噻二唑类化合物的合成和性质研究
}

\author{
任亚平 $a$ 刘 絮 $a$ 王瑞 $b$ 周元清 $a$ \\ 李 标 $a$ 徐 琰*, $a$ 宋毛平 $a$ \\ ( ${ }^{a}$ 郑州大学化学与分子工程学院 郑州 450001) \\ $\left({ }^{b}\right.$ 定州英才实验中学 定州 073000)
}

\begin{abstract}
摘要 以对二茂铁苯甲酸和 2-氨基-5-芳基-1,3,4-噻二唑化合物为原料, 合成了 7 个新的二茂铁苯甲酰噻二唑化合物 $\mathrm{FcL}_{1} \sim \mathrm{FcL}_{7}$. 采用 IR、 ${ }^{1} \mathrm{H} \mathrm{NMR}$ 和元素分析对化合物进行了表征. 用 X-Ray 单晶衍射测定了化合物 $\mathrm{FcL}_{4}$ 的晶体结构. 电化学研究表明, 化合物在电极表面发生可逆的单电子转移, $\mathrm{FcL}_{1} \sim \mathrm{FcL}_{7}$ 对 $\mathrm{Pb}^{2+}$ 和 $\mathrm{Zn}^{2+}$ 都有一定的电化学响应. 抗菌 活性试验表明 $\mathrm{FcL}_{1} \sim \mathrm{FcL}_{7}$ 均对玉米禾谷镰刀菌有较好的抑菌活性和选择性. 抗癌活性试验表明 $\mathrm{FcL}_{5} \sim \mathrm{FcL}_{7}$ 对人体食管 癌细胞的抑制作用明显.
\end{abstract}

关键词 二茂铁; 噻二唑; 单晶结构; 电化学性质; 生物活性

\section{Synthesis and Properties of 4-Ferrocenyl-benzoyl-thiadiazole Derivatives}

\author{
Ren, Yaping ${ }^{a} \quad \mathrm{Liu}, \mathrm{Xu}^{a} \quad$ Wang, Rui $^{b} \quad$ Zhou, Yuanqing $^{a}$ \\ $\mathrm{Li}, \mathrm{Biao}^{a} \quad \mathrm{Xu}, \mathrm{Yan}^{*, a}$ Song, Maoping ${ }^{a}$ \\ ( ${ }^{a}$ College of Chemistry and Molecular Engineering, Zhengzhou University, Zhengzhou 450001) \\ ( ${ }^{b}$ Dingzhou Yingcai Middle School, Dingzhou 073000)
}

\begin{abstract}
Seven kinds of ferrocenyl-benzoyl-thiadiazole compounds $\mathrm{FcL}_{1} \sim \mathrm{FcL}_{7}$ were synthesized by using 4-ferrocenylbenzoic and 2-amino-5-aryl-1,3,4-thiadiazole as raw materials. These compounds were characterized by IR, ${ }^{1} \mathrm{H}$ NMR and elemental analysis. The crystal structure of $\mathrm{FcL}_{4}$ was determined by X-ray diffraction analysis. The electrochemical research showed that the redox reaction on the surface of electrode was reversible with single electron, and $\mathrm{FcL}_{1} \sim \mathrm{FcL}_{7}$ have a certain redox response to $\mathrm{Pb}^{2+}$ and $\mathrm{Zn}^{2+}$. The antibacterial activity tests indicated that $\mathrm{FcL}_{1} \sim \mathrm{FcL}_{7}$ presented significant activity and selectivity against Fusarium graminearum. $\mathrm{FcL}_{5} \sim \mathrm{FcL}_{7}$ exhibited good inhibition against human esophageal cancer cells in the anticancer activity tests.
\end{abstract}

Keywords ferrocene; thiadiazole; crystal structure; electrochemistry; biological activity

噻二唑类化合物是一种易形成氢键结合域、含 “N-C-S” 的药效活性基团 ${ }^{[1]}$, 是生产常见抗生素如头孢 唑啉、头孢西酮、头孢卡奈、头孢帕罗、唑酮头孢菌素 等医药的重要中间体 ${ }^{[2]} .1,3,4$-噻二唑具有良好的电子传 输水平和空穴阻挡能力 ${ }^{[3]}$, 其特殊的结构单元能与生物 体内某些金属离子配位, 具有较好的组织细胞通透性 ${ }^{[4]}$. 近几年来研究发现, 2,5-二取代-1,3,4-噻二唑类衍生物具 有抗癌 ${ }^{[5,6]}$ 、抑制阿尔茨海默疾病 ${ }^{[7]}$ 、消炎 ${ }^{[8]}$ 、抗惊厥 ${ }^{[9]}$ 等多种生物活性. 将不同的杂环引入到 1,3,4-噻二唑环
上 2 位或 5 位, 会使噻二唑活性增强, 并可明显增加其 药性 ${ }^{[10,11]}$. 因此, 对于 2,5-二取代-1,3,4-噻二㭫衍生物的 合成与性能研究已受到人们广泛的关注.

二茂铁衍生物能在多数常见溶剂中进行可逆的单 电子氧化还原反应, 是一种较好的电子转移媒介 ${ }^{[12]}$.二 茂铁衍生物的低毒性、亲油性使其在抗菌 ${ }^{[13]}$ 、抗肿 瘤 ${ }^{[14,15]}$ 药物的研发方面得到广泛研究和应用. 将二茂铁 和噻二唑结合得到的化合物具有可逆的氧化还原性 ${ }^{[16]}$, 能与金属离子络合, 应用于离子选择性电极 ${ }^{[17]}$; 还具有

\footnotetext{
* Corresponding author. E-mail: xuyan@zzu.edu.cn

Received July 5, 2016; revised September 6, 2016; published online September 19, 2016.

Project supported by the National Natural Science Foundation of China (No. 21171149).

国家自然科学基金(No. 21171149)资助项目.
} 
优良的生物活性 ${ }^{[18]}$ 、抗菌活性 ${ }^{[19,20]}$ 等. 二茂铁苯甲酸具 有多个活性中心和扩展的共轭面, 有利于分子间的一些 弱相互作用 ${ }^{[21]}$. 冯志君等 ${ }^{[22]}$ 合成了一系列二茂铁苯甲 酰塞二唑类化合物, 但没有对其性质进行深入的研究. 在此, 我们将二茂铁苯甲酸与 1,3,4-噻二唑衍生物结合, 分别引入了供电子基团和吸电子基团, 增加了杂原子的 数量, 期望得到活性更好的化合物. 本文得到了 7 个新 的二茂铁苯甲酰噻二唑化合物, 对它们的结构进行了表 征, 并研究了 7 种化合物电化学性质和生物活性. 其合 成路线见 Scheme 1.

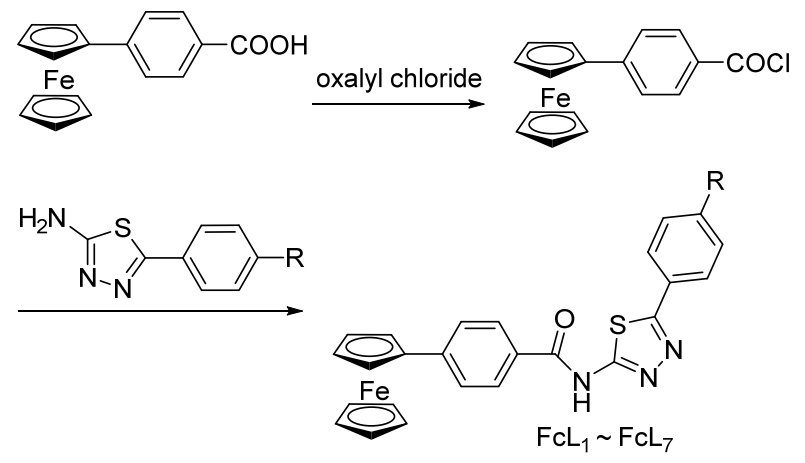

$\mathrm{FcL}_{1}: \mathrm{R}=\mathrm{OCH}_{3} ; \mathrm{FcL}_{2}: \mathrm{R}=\mathrm{CH}_{3} ; \mathrm{FcL}_{3}: \mathrm{R}=\mathrm{H} ; \mathrm{FcL}_{4}: \mathrm{R}=\mathrm{Cl}$; $\mathrm{FcL}_{5}: \mathrm{R}=\mathrm{Br} ; \mathrm{FcL}_{6}: \mathrm{R}=\mathrm{NO}_{2} ; \mathrm{FcL}_{7}: \mathrm{R}=\mathrm{CF}_{3}$

图式 1 化合物 $\mathrm{FeL}_{1} \sim \mathrm{FeL}_{7}$ 的合成

Scheme 1 Synthesis of compounds $\mathrm{FeL}_{1} \sim \mathrm{FeL}_{7}$

\section{1 结果与讨论}

\section{1 目标化合物 $\mathrm{FCL}_{4}$ 晶体结构解析及结果}

化合物 $\mathrm{FcL}_{4}\left(\mathrm{C}_{27} \mathrm{H}_{24} \mathrm{ClFeN}_{3} \mathrm{O}_{2} \mathrm{~S}_{2}\right)$ 为梭形块状深红色 晶体, 分子晶体图如图 1 (CCDC: 1484853). 单晶 $\mathrm{X}$ 射线 测试表明化合物 $M=577.91$, 属于三斜晶系, $P \overline{1}$ 手性空 间群. 晶胞参数: $a=8.6731(4) \AA, b=12.0650(6) \AA, c=$ 12.9753(7) $\AA, \alpha=82.583(2)^{\circ}, \quad \beta=73.951(2)^{\circ}, \quad \gamma=$ $71.962(2)^{\circ}, V=1239.29(11) \AA^{3}, D_{\mathrm{c}}=1.549 \mathrm{Mg} / \mathrm{m}^{3}$, 晶胞 中分子数: $Z=2, F(000)=596$, 吸收系数 $\mu=0.917$ $\mathrm{mm}^{-1}, R_{1}=0.0348[I>2 \sigma(I)], w R_{2}=0.1111, s=0.914$.

晶体结构数据表明, $\mathrm{FcL}_{4}$ 中 $\mathrm{Fe}-\mathrm{C}$ 键长范围为 $2.033 \sim 2.054 \AA$, 两个茂环面间距为 $3.297 \AA$, 由于苯环 的取代, 茂环发生扭曲, 二面角为 $3.414^{\circ}$. 茂环与其直 接相连的苯环共平面, 与噻二唑环的夹角为 $11.442^{\circ}$, 与 末端苯环的二面角为 $16.948^{\circ}$, 呈现逐渐偏转的构型, 噻 二唑环和末端苯环上的原子偏离其平面的平均距离分 别为 0.005 和 $0.008 \AA$. 羰基氧原子与茂环上的氢形成分 子间的氢键 $\mathrm{C}(5)-\mathrm{H}(14) \cdots \mathrm{O}(1)$, 间距为 $2.661 \AA$, 键角 为 $157.498^{\circ}$. 此外, 溶剂分子 DMSO 上的氧原子分别与 两个不同 $\mathrm{FcL}_{4}$ 分子上茂环上的氢和酰胺上氮原子形成 分子间氢键: $\mathrm{C}(25)-\mathrm{H}(16) \cdots \mathrm{O}(2)(2.712 \AA)$ 以及 $\mathrm{N}(1)-$
$\mathrm{H}(14) \cdots \mathrm{O}(2)(2.050 \AA)$. 分子间具有共轭体系的苯环和 噻二唑环之间还存在 $\pi \cdots \pi$ 堆积, 构成三维堆积的孔洞 结构(图 2).

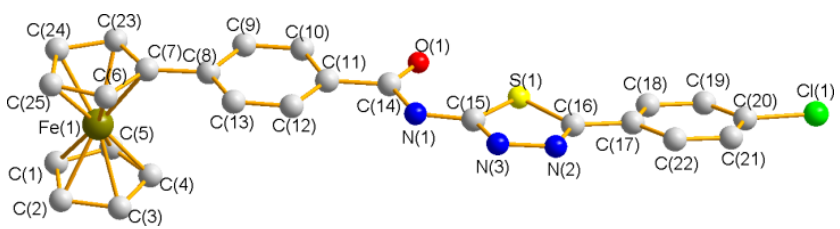

图 $1 \mathrm{FcL}_{4}$ 的分子结构

Figure 1 Molecular structure of $\mathrm{FcL}_{4}$

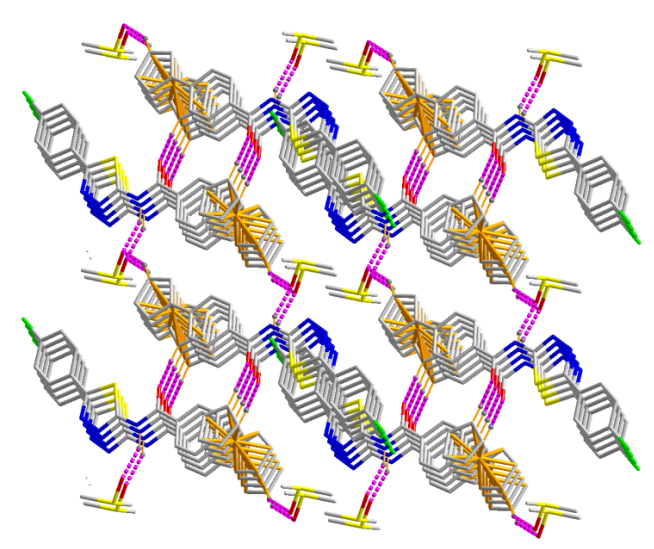

图 $2 \mathrm{FcL}_{4}$ 的分子间氢键和 $\pi \cdots \pi$ 作用堆积图

Figure 2 3D molecular structure of hydrogen bonds and $\pi \cdots \pi$ stacking in the $\mathrm{FcL}_{4}$

\section{2 电化学性质}

\subsection{1 循环伏安}

目标化合物的循环伏安数据见表 $1 . \mathrm{FcL}_{1} \sim \mathrm{FcL}_{7}$ 的 电化学性质相似, 在 $0.3 \sim 0.9 \mathrm{~V}$ 间均有一对对称的氧化 还原峰, $i_{\mathrm{pa}} / i_{\mathrm{pc}}$ 接近 1 , 初步判定电极表面发生了单电子 的氧化还原反应，可归属为 $\mathrm{Fc}^{+} / \mathrm{Fc}\left(\mathrm{Fe}^{3+}+\mathrm{e} \leftrightharpoons \mathrm{Fe}^{2+}\right)$ 的氧 化还原. 化合物半波电位很接近但相比二茂铁都有了明 显的正移，说明二茂铁上取代基的引入，整个分子的体 积增大，茂环难以靠近电极表面，使氧化还原峰发生正 移 ${ }^{[23]}$. 进一步改变扫描速率, 得到 $\mathrm{FcL}_{1} \sim \mathrm{FcL}_{7}$ 在不同扫 速下的循环伏安图, 作 $i_{p} \sim v^{1 / 2}$ 图. 以 $\mathrm{FcL}_{1}$ 为例(图 3), 随 着扫描速率的升高, 氧化还原峰电位没有明显变化, $|\Delta E|$ 基本不变，说明在扫描速率 $50 \sim 500 \mathrm{mV} / \mathrm{s}$ 的范围 内, 目标化合物在电极上发生的氧化还原过程是准可逆 的. $i_{p} \sim v^{1 / 2}$ 呈线性关系, 表明化合物的氧化还原电对在 电极表面的反应过程是受扩散控制的. 循环伏安数据见 表 1 .

\subsection{2 离子响应}

进一步测试了化合物 $\mathrm{FcL}_{1} \sim \mathrm{FcL}_{7}$ 对金属离子的响 应, 结果见表 2. 当分别加入 $\mathrm{Fe}^{2+} 、 \mathrm{Cd}^{2+} 、 \mathrm{Hg}^{2+} 、 \mathrm{Mn}^{2+}$ 、 $\mathrm{Co}^{2+} 、 \mathrm{Ni}^{2+} 、 \mathrm{Ag}^{+}$时, 化合物 $\mathrm{FcL}_{1} \sim \mathrm{FcL}_{7}$ 的电位差较小, 
表 1 化合物 $\mathrm{FcL}_{1} \sim \mathrm{FcL}_{7}$ 的循环伏安数据 ${ }^{a}$

Table 1 The electrochemical data of $\mathrm{FcL}_{1} \sim \mathrm{FcL}_{7}$

\begin{tabular}{cccccc}
\hline Compd. & $E_{\mathrm{pa}} / \mathrm{V}$ & $E_{\mathrm{pc}} / \mathrm{V}$ & $E^{0} / \mathrm{V}$ & $\Delta E / \mathrm{V}$ & $i_{\mathrm{pa}} / i_{\mathrm{pc}}$ \\
\hline $\mathrm{Fc}$ & 0.537 & 0.471 & 0.504 & 0.066 & 1.0000 \\
$\mathrm{FcL}_{1}$ & 0.650 & 0.576 & 0.613 & 0.074 & 1.1765 \\
$\mathrm{FcL}_{2}$ & 0.638 & 0.580 & 0.609 & 0.058 & 1.3384 \\
$\mathrm{FcL}_{3}$ & 0.636 & 0.575 & 0.605 & 0.061 & 1.2773 \\
$\mathrm{FcL}_{4}$ & 0.634 & 0.570 & 0.602 & 0.064 & 1.2623 \\
$\mathrm{FcL}_{5}$ & 0.617 & 0.553 & 0.585 & 0.064 & 1.2742 \\
$\mathrm{FcL}_{6}$ & 0.616 & 0.547 & 0.581 & 0.069 & 1.3938 \\
$\mathrm{FcL}_{7}$ & 0.631 & 0.579 & 0.605 & 0.052 & 1.3793 \\
\hline
\end{tabular}

${ }^{a} E_{\mathrm{pa}}$ : 阳极峰电位, $E_{\mathrm{pc}}$ : 阴极峰电位, $E^{0}:$ 半波电位, $i_{\mathrm{pa}}$ : 阳极峰电流, $i_{\mathrm{pc}}$ : 阴 极峰电流。

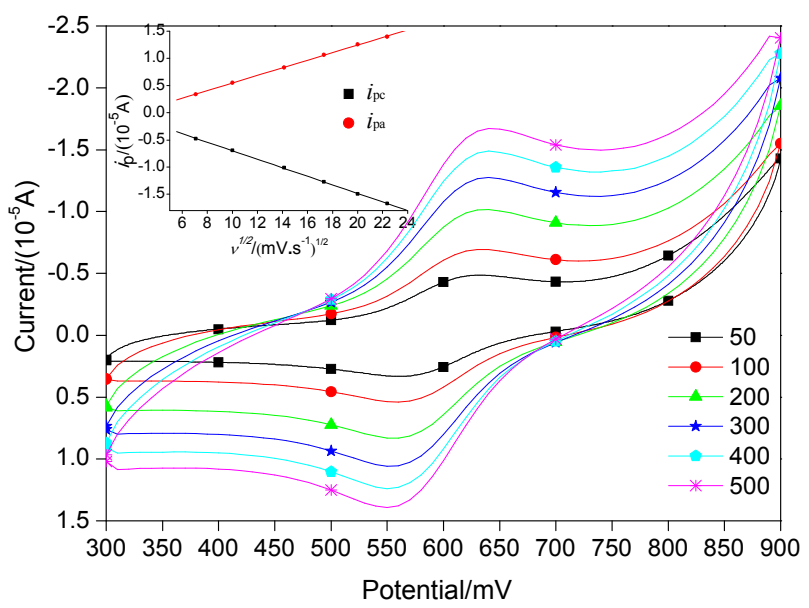

图 $3 \mathrm{FcL}_{1}$ 在 $\mathrm{DMF}$ 中不同扫速下的循环伏安图以及 $i_{\mathrm{p}} \sim v^{1 / 2}$ 关系图

Figure $3 \mathrm{CVs}$ of $\mathrm{FcL}_{1}$ in DMF at different scan rate and the relation between $i_{\mathrm{p}} \sim v^{1 / 2}$

表 2 化合物 $\mathrm{FcL}_{1} \sim \mathrm{FcL}_{7}$ 对 $\mathrm{Pb}^{2+}$ 和 $\mathrm{Zn}^{2+}$ 的电化学响应 Table 2 Electrochemical responds of $\mathrm{FcL}_{1} \sim \mathrm{FcL}_{7}$ to $\mathrm{Pb}^{2+}$ and $\mathrm{Zn}^{2+}$

\begin{tabular}{ccccccc}
\hline \multirow{2}{*}{ Compd. } & $E_{\mathrm{pa}} / \mathrm{mV}$ & \multicolumn{2}{c}{$E_{\mathrm{pa}} / \mathrm{mV}$} & & \multicolumn{2}{c}{$\Delta E_{\mathrm{pa}} / \mathrm{mV}$} \\
\cline { 7 - 8 } \cline { 6 - 7 } & & $\left(\mathrm{Pb}^{2+}+\mathrm{L}\right)$ & $\left(\mathrm{Zn}^{2+}+\mathrm{L}\right)$ & & $\left(\mathrm{Pb}^{2+}+\mathrm{L}\right)$ & $\left(\mathrm{Zn}^{2+}+\mathrm{L}\right)$ \\
\hline $\mathrm{FcL}_{1}$ & 621 & 581 & 572 & & 40 & 49 \\
$\mathrm{FcL}_{2}$ & 620 & 579 & 572 & & 41 & 48 \\
$\mathrm{FcL}_{3}$ & 621 & 574 & 578 & & 47 & 43 \\
$\mathrm{FcL}_{4}$ & 606 & 563 & 561 & & 43 & 45 \\
$\mathrm{FcL}_{5}$ & 607 & 558 & 565 & & 49 & 42 \\
$\mathrm{FcL}_{6}$ & 613 & 577 & 575 & & 36 & 38 \\
$\mathrm{FcL}_{7}$ & 626 & 565 & 570 & & 61 & 56 \\
\hline
\end{tabular}

但当加入 $\mathrm{Pb}^{2+} 、 \mathrm{Zn}^{2+}$ 离子时, 目标化合物的氧化还原峰 均发生了一定程度的移动, 且电位差值大于 $25 \mathrm{mV}$, 即 目标化合物 $\mathrm{FcL}_{1} \sim \mathrm{FcL}_{7}$ 对 $\mathrm{Pb}^{2+} 、 \mathrm{Zn}^{2+}$ 金属离子具有一 定的电化学响应能力, 其中 $\mathrm{FcL}_{7}$ 与 $\mathrm{Pb}^{2+} 、 \mathrm{Zn}^{2+}$ 的响应最 为明显. 化合物与金属离子作用前后电位值改变越大, 说明发生氧化还原反应所需的能量越高, 络合形成的键 就越强, 它们相互作用形成络合物的稳定性就越高, 由
此推断, $\mathrm{FcL}_{7}$ 与 $\mathrm{Pb}^{2+} 、 \mathrm{Zn}^{2+}$ 形成的配合物稳定性最强.

\section{3 生物活性}

\subsection{1 抗菌活性的测定}

采用平血生长速率法，对目标化合物的植物病原菌 抑菌活性进行研究。供试菌种为玉米禾谷镰刀菌 (Fusarium graminearum)、玉米新月弯狍菌(Curvularia lunata)、链格孢菌(Alternaria tenuis). 结果见表 3.

表 3 化合物 $\mathrm{FcL}_{1} \sim \mathrm{FcL}_{7}$ 对三种细菌的抑制活性(抑制率/\%)

Table 3 Antibacterial activities of $\mathrm{FcL}_{1} \sim \mathrm{FcL}_{7}$ (inhibition rate $/ \%)$

\begin{tabular}{cccc}
\hline Compd. & 玉米禾谷镰刀菌 & 新月弯孢菌 & 链格狍菌 \\
\hline $\mathrm{FcL}_{1}$ & 63.42 & 24.37 & 43.55 \\
$\mathrm{FcL}_{2}$ & 68.37 & 22.63 & 46.05 \\
$\mathrm{FcL}_{3}$ & 72.58 & 34.79 & 52.94 \\
$\mathrm{FcL}_{4}$ & 75.30 & 37.81 & 49.41 \\
$\mathrm{FcL}_{5}$ & 79.23 & 42.51 & 55.61 \\
$\mathrm{FcL}_{6}$ & 82.37 & 39.42 & 53.39 \\
$\mathrm{FcL}_{7}$ & 84.25 & 43.58 & 56.24 \\
\hline
\end{tabular}

测试浓度为 $200 \mu \mathrm{g} / \mathrm{mL}$.

从表 3 可以看出, 目标化合物对这三种植物病原菌 均有不同程度的抑菌活性. 尤其对玉米禾谷镰刀菌的抑 制作用最为明显, 对链格狍菌和新月弯狍菌的抑制几乎 不明显, 说明其抗菌活性具有一定的选择性. 而 $\mathrm{CF}_{3}$ 为 取代基的化合物对三种植物病原菌都有一定的抑制作 用. 对于玉米禾谷镰刀菌来说, $\mathrm{FcL}_{7}$ 的抑制率最高. 且 随着取代基的不同，化合物对玉米禾谷镰刀菌抑菌活性 也不尽相同, 以 $\mathrm{NO}_{2} 、 \mathrm{CF}_{3}$ 为取代基的抑菌效果明显, 抑 制率达到 $80 \%$ 以上. 不难发现, $\mathrm{FcL}_{7}$ 对三种植物病原菌 的抑制作用都比其他几种化合物的抑制作用好，可能归 因于 $\mathrm{CF}_{3}$ 的强吸电子能力. 初步进行构效分析得出, 化 合物的抑菌性与 $\mathrm{R}$ 取代基的吸电子能力有关, 即 $\mathrm{R}$ 的吸 电子能力越强, 化合物对植物病原菌的抑制作用越明 显.

对化合物 $\mathrm{FcL}_{1} \sim \mathrm{FcL}_{7}$ 对玉米禾谷镰刀菌进行 $\mathrm{EC}_{50}$ 值测定并计算毒力回归方程, 结果见表 4. 从表中可以 更准确地看出, 目标化合物对玉米禾谷镰刀菌的抑制作 用良好，其中 $\mathrm{FcL}_{6} 、 \mathrm{FcL}_{7}$ 对玉米禾谷镰刀菌的 $\mathrm{EC}_{50}$ 值 分别为 98.92 和 $100.30 \mu \mathrm{g} / \mathrm{mL}$. 这两种化合物可以作为 先导物质进行进一步的结构优化研究.

\subsection{2 抗肿瘤活性的测定}

本实验采用 CCK-8 法, 在五种浓度梯度下测试了 目标化合物对人胃癌细胞株(BGC-823)、人胃癌细胞株 (SGC-7901)、人食管癌细胞株(EC-9706)、人食管癌细胞 株(Eca-109)、人食管癌细胞株(Ece)、白血病细胞株 (Jurkat)的抗癌活性. 结果见表 5. 
表 4 化合物 $\mathrm{FcL}_{1} \sim \mathrm{FcL}_{7}$ 对玉米禾谷镰刀菌的抑制效果

Table 4 The inhibitory effect of $\mathrm{FcL}_{1} \sim \mathrm{FcL}_{7}$ against Fusarium graminearum

\begin{tabular}{cccc}
\hline Compd. & Toxic regression equation & $r$ & $\mathrm{EC}_{50} /\left(\mu \mathrm{g} \bullet \mathrm{mL}^{-1}\right)$ \\
\hline $\mathrm{FcL}_{1}$ & $y=2.0955 x+0.4159$ & 0.9676 & 154.03 \\
$\mathrm{FcL}_{2}$ & $y=2.6316 x-0.5953$ & 0.9986 & 133.72 \\
$\mathrm{FcL}_{3}$ & $y=2.2955 x+0.1843$ & 0.9751 & 125.28 \\
$\mathrm{FcL}_{4}$ & $y=2.6606 x-0.6199$ & 0.9668 & 129.50 \\
$\mathrm{FcL}_{5}$ & $y=2.8025 x-0.7432$ & 0.9847 & 112.02 \\
$\mathrm{FcL}_{6}$ & $y=2.7004 x-0.3881$ & 0.9904 & 98.92 \\
$\mathrm{FcL}_{7}$ & $y=2.8092 x-0.6221$ & 0.9764 & 100.30 \\
\hline
\end{tabular}

表 5 化合物 $\mathrm{FcL}_{1} \sim \mathrm{FcL}_{7}$ 对癌细胞株的 $\mathrm{IC}_{50}(\mu \mathrm{mol} / \mathrm{L})$ 值 ${ }^{a}$

Table $5 \quad \mathrm{IC}_{50}(\mu \mathrm{mol} / \mathrm{L})$ of $\mathrm{FcL}_{1} \sim \mathrm{FcL}_{7}$ for colibacillus

\begin{tabular}{ccccccc}
\hline Compd. & BGC-823 & SGC7901 & EC-9706 & Eca-109 & Ece & Jurkat \\
\hline FcL $_{1}$ & $\mathrm{~N}$ & $\mathrm{~N}$ & $\mathrm{~N}$ & $\mathrm{~N}$ & $\mathrm{~N}$ & $\mathrm{~N}$ \\
$\mathrm{FcL}_{2}$ & $\mathrm{~N}$ & $\mathrm{~N}$ & 87.99 & $\mathrm{~N}$ & $\mathrm{~N}$ & $\mathrm{~N}$ \\
$\mathrm{FcL}_{3}$ & $\mathrm{~N}$ & $\mathrm{~N}$ & $\mathrm{~N}$ & $\mathrm{~N}$ & $\mathrm{~N}$ & $\mathrm{~N}$ \\
$\mathrm{FcL}_{4}$ & $\mathrm{~N}$ & $\mathrm{~N}$ & $\mathrm{~N}$ & $\mathrm{~N}$ & 85.41 & $\mathrm{~N}$ \\
$\mathrm{FcL}_{5}$ & $\mathrm{~N}$ & 80.11 & 44.23 & 41.28 & $\mathrm{~N}$ & $\mathrm{~N}$ \\
$\mathrm{FcL}_{6}$ & $\mathrm{~N}$ & $\mathrm{~N}$ & 71.67 & 18.42 & $\mathrm{~N}$ & $\mathrm{~N}$ \\
$\mathrm{FcL}_{7}$ & $\mathrm{~N}$ & $\mathrm{~N}$ & 19.31 & 24.91 & $\mathrm{~N}$ & $\mathrm{~N}$ \\
\hline
\end{tabular}

${ }^{a} \mathrm{~N}$ 表示 $\mathrm{IC}_{50}$ 值大于 100 , 没有活性.

由表 5 可知，当取代基为 $\mathrm{CH}_{3}, \mathrm{H}$ 时,目标化合物 $\mathrm{FcL}_{1} 、 \mathrm{FcL}_{3}$ 对六种被测的肿瘤细胞株均未呈现出明显的 抑制活性. $\mathrm{FcL}_{2}$ 和 $\mathrm{FcL}_{4}$ 对人食管癌细胞株 EC-9706 和 Ece 的抑制作用较弱. 而当在二茂铁苯甲酰噻二唑骨架 上引入强吸电子基团时, 化合物 $\mathrm{FcL}_{5} \sim \mathrm{FcL}_{7}$ 对于人食 管癌细胞 EC-9706 和 Eca-109 的抑制作用明显提高. 其 中取代基为 $\mathrm{CF}_{3}$ 的化合物 $\mathrm{FcL}_{7}$ 对 EC-9706 的抑制性较 好, 其 $\mathrm{IC}_{50}$ 值为 $19.31 \mu \mathrm{mol} / \mathrm{L}$. 取代基为 $\mathrm{NO}_{2}$ 的化合物 $\mathrm{FcL}_{6}$ 对于 Eca-109 的抑制性较好, 其 $\mathrm{IC}_{50}$ 值是 18.42 $\mu \mathrm{mol} / \mathrm{L}$. 结合化合物结构与抗肿瘤活性来看, 随着取代 基的吸电子能力逐渐增强, 其 $\mathrm{IC}_{50}$ 值也显著地减小, 即 抗肿瘤活性在逐渐增强, 说明当 $\mathrm{R}$ 是吸电子基团时更有 利于化合物抗肿瘤活性的表达.

\section{2 结论}

本文设计合成了 7 种新型对二茂铁苯甲酰噻二唑类 化合物, 得到了化合物 $\mathrm{FcL}_{4}$ 的单晶结构. 电化学实验表 明，该类化合物在电极表面都发生了可逆的单电子氧化 还原反应, 且该类化合物对 $\mathrm{Pb}^{2+} 、 \mathrm{Zn}^{2+}$ 有一定的电化学 响应能力. 生物活性测试表明, $\mathrm{R}$ 取代基为吸电子基团 时有利于其杀菌活性和抗癌活性, 7 种化合物对玉米禾 谷镰刀菌都有较好的抑菌活性和选择性, $\mathrm{FcL}_{7}$ 在浓度为 $200 \mu \mathrm{g} / \mathrm{mL}$ 时对玉米禾谷镰刀菌的抑制率为 $84.25 \%$; $\mathrm{FcL}_{5} \sim \mathrm{FcL}_{7}$ 对人体食管癌细胞抑制作用明显, $\mathrm{FcL}_{6}$ 对人 体食管癌细胞 Eca-109 的 $\mathrm{IC}_{50}$ 值为 $18.42 \mu \mathrm{mol} / \mathrm{L}$. 为新 的具有电化学活性和生物活性的化合物的合成、篮选、
开发提供一些基本的理论数据和参考.

\section{3 实验部分}

\section{1 仪器与试剂}

AVIII HD 600 Bruker 核磁共振仪; Flash EA 1112 型 元素分析仪; 美国 Nicolet 470-FTIR 红外光谱仪; 德国 NETZSCH STA 409 PC 同步热分析仪; 郑州远大科技有 限公司 RST3000 电化学工作站. 所用试剂均为市售化 学纯或分析纯, 使用前经过干燥处理.

\section{2 实验方法}

\section{2 .1 对二茂铁苯甲酸的合成}

对二茂铁苯甲酸参照文献[24]方法合成.

\subsubsection{2-氨基-5-芳基-1,3,4-噻二唑衍生物的合成}

2-氨基-5-芳基-1,3,4-噻二唑衍生物照文献[25]方法 合成.

\subsection{3 目标化合物的合成}

称取 $2 \mathrm{mmol}(0.612 \mathrm{~g})$ 对二茂铁苯甲酸溶于 $50 \mathrm{~mL}$ 的无水氯仿中, 缓慢滴加 $4 \mathrm{~mL}$ 新蒸的草酰氯, 在 $60{ }^{\circ} \mathrm{C}$ 下回流搅拌 $4 \mathrm{~h}$. 反应完后, 旋蒸除去溶剂和未完全反 应的草酰氯，旋干后得到深红色小方片状固体二茂铁苯 甲酰氯.

称取 $2 \mathrm{mmol}(0.354 \mathrm{~g})$ 5-苯基-2-氨基-1,3,4-噻二唑 溶于 $80 \mathrm{~mL}$ 的无水二氯甲烷中, 滴加 $2 \mathrm{~mL}$ 的重蒸过 的三乙胺作为缚酸剂，将新制得的二茂铁苯甲酰氯溶 于 $50 \mathrm{~mL}$ 的二氯甲烷中, 缓慢滴加到上述反应体系内, 搅拌回流, TLC 监测, 至反应完成. 将反应液依次用饱 和碳酸氢钠溶液、蒸馏水、饱和食盐水洗涤，收集有机 相, 加入无水硫酸镁干燥, 过滤, 将滤液浓缩, 采用柱 层析分离提纯得到目标产物.

$N$-[5-(4-甲氧基苯基)-1,3,4-噻二唑-2-基]-4-二茂铁 苯甲酰胺 $\left(\mathrm{FcL}_{1}\right)$ : 展开剂: $V($ 乙酸乙酯 $): V($ 石油醚 $)=$ 1：1, 橘红色固体, 产率 46\%. m.p. $246 \sim 248{ }^{\circ} \mathrm{C} ;{ }^{1} \mathrm{H}$ NMR (DMSO- $\left.d_{6}, 600 \mathrm{MHz}\right) \delta: 13.00(\mathrm{~s}, 1 \mathrm{H}, \mathrm{H}-\mathrm{N}), 8.10$ (d, $J=8.5 \mathrm{~Hz}, 2 \mathrm{H}, \mathrm{H}-\mathrm{Ar}$ ), 7.89 (d, $J=8.1 \mathrm{~Hz}, 2 \mathrm{H}, \mathrm{H}-\mathrm{Ar}$ ), 7.73 (d, $J=8.5 \mathrm{~Hz}, 2 \mathrm{H}, \mathrm{H}-\mathrm{Ar}), 7.37$ (d, $J=8.1 \mathrm{~Hz}, 2 \mathrm{H}$, H-Ar), 4.96 (s, J=1.8 Hz, 2H, H-Fc), 4.46 (s, 2H, H-Fc), 4.05 (s, 5H, H-Fc), 3.85 (s, 3H, $\left.\mathrm{H}-\mathrm{OCH}_{3}\right)$; IR (KBr) v: 3156, 3044, 2944, 1669, 1617, 1519, 1303, 1249, 1115, $1043,821,695 \mathrm{~cm}^{-1}$. Anal. calcd for $\mathrm{C}_{26} \mathrm{H}_{21} \mathrm{FeN}_{3} \mathrm{O}_{2} \mathrm{~S}: \mathrm{C}$ 63.04, N 8.48, H 4.27, S 6.47; found C 62.16, N 8.17, H 4.49, S 6.38 .

$N$-[5-(4-甲基苯基)-1,3,4-噻二唑-2-基]-4-二茂铁苯 甲酰胺 $\left(\mathrm{FcL}_{2}\right)$ : 展开剂: $V($ 乙酸乙酯 $): V($ 石油醚 $)=1: 1$, 橘红色固体，产率 37\%. m.p. 273 274 ${ }^{\circ} \mathrm{C} ;{ }^{1} \mathrm{H} \mathrm{NMR}$ 
(DMSO- $\left.d_{6}, 600 \mathrm{MHz}\right) \delta: 13.06$ (s, 1H, H-N), 8.10 (d, $J=$ $8.5 \mathrm{~Hz}, 2 \mathrm{H}, \mathrm{H}-\mathrm{Ar}$ ), 7.89 (d, J=8.1 Hz, 2H, H-Ar), 7.73 (d, $J=8.5 \mathrm{~Hz}, 2 \mathrm{H}, \mathrm{H}-\mathrm{Ar}$ ), 7.37 (d, $J=8.1 \mathrm{~Hz}, 2 \mathrm{H}, \mathrm{H}-\mathrm{Ar}$ ), 4.97 (s, $J=1.8 \mathrm{~Hz}, 2 \mathrm{H}, \mathrm{H}-\mathrm{Fc}$ ), 4.47 (s, 2H, H-Fc), 4.08 (s, 5H, $\mathrm{H}-\mathrm{Fc}$ ), 2.39 (s, 3H, $\mathrm{H}-\mathrm{CH}_{3}$ ); IR (KBr) v: 3112, 3010, 2932, 1659, 1591, 1525, 1313, 1223, 1111, 1099, 819, $697 \mathrm{~cm}^{-1}$. Anal. calcd for $\mathrm{C}_{26} \mathrm{H}_{21} \mathrm{FeN}_{3} \mathrm{OS}$ : C 65.14, N 8.77, H 4.42, S 6.69; found C 64.98, N 8.75, H 4.54, S 6.65 .

$N$-[5-(4-苯基)-1,3,4-噻二唑-2-基]-4-二茂铁苯甲酰 胺 $\left(\mathrm{FcL}_{3}\right)$ : 展开剂: $V($ 乙酸乙酯 $): V($ 石油醚 $)=1: 1$, 砖 红色固体, 产率 30\%. m.p. $261 \sim 263{ }^{\circ} \mathrm{C} ;{ }^{1} \mathrm{H}$ NMR (DMSO- $\left.d_{6}, 600 \mathrm{MHz}\right) \delta: 13.13$ (s, $\left.1 \mathrm{H}, \mathrm{H}-\mathrm{N}\right), 8.10$ (d, $J=$ $8.5 \mathrm{~Hz}, 2 \mathrm{H}, \mathrm{H}-\mathrm{Ar}$ ), 8.00 (s, $J=8.1 \mathrm{~Hz}, 2 \mathrm{H}, \mathrm{H}-\mathrm{Ar}$ ), 7.73 (d, $J=8.5 \mathrm{~Hz}, 2 \mathrm{H}, \mathrm{H}-\mathrm{Ar}$ ), 7.57 (s, $J=8.1 \mathrm{~Hz}, 3 \mathrm{H}, \mathrm{H}-\mathrm{Ar}$ ), 4.97 (s, $J=1.8 \mathrm{~Hz}, 2 \mathrm{H}, \mathrm{H}-\mathrm{Fc}$ ), 4.47 (s, 2H, H-Fc), 4.05 (s, 5H, H-Fc); IR (KBr) v: 3152, 3023, 2925, 1664, 1607, 1519, $1421,1309,1187,1090,823,759,686 \mathrm{~cm}^{-1}$. Anal. calcd for $\mathrm{C}_{25} \mathrm{H}_{19} \mathrm{FeN}_{3} \mathrm{OS}$ : C 64.53, N 9.03, H 4.12, $\mathrm{S}$ 6.89; found C 64.56, N 8.81, H 4.20, S 6.81.

$N$-[5-(4-氯基苯基)-1,3,4-噻二唑-2-基]-4-二茂铁苯 甲酰胺 $\left(\mathrm{FcL}_{4}\right)$ : 展开剂: $V$ (二氯甲烷) $: V$ (甲醇 $)=20 ： 1$, 红色固体, 产率 $22 \%$. m.p. $220 \sim 223{ }^{\circ} \mathrm{C} ;{ }^{1} \mathrm{H}$ NMR (DMSO- $\left.d_{6}, 600 \mathrm{MHz}\right) \delta$ : 13.12 (s, 1H, H-N), 8.10 (d, $J=$ $8.5 \mathrm{~Hz}, 2 \mathrm{H}, \mathrm{H}-\mathrm{Ar}$ ), 8.02 (s, $J=8.1 \mathrm{~Hz}, 2 \mathrm{H}, \mathrm{H}-\mathrm{Ar}$ ), 7.73 (d, $J=8.5 \mathrm{~Hz}, 2 \mathrm{H}, \mathrm{H}-\mathrm{Ar}$ ), 7.63 (s, $J=8.1 \mathrm{~Hz}, 3 \mathrm{H}, \mathrm{H}-\mathrm{Ar}$ ), 4.96 (s, $J=1.8 \mathrm{~Hz}, 2 \mathrm{H}, \mathrm{H}-\mathrm{Fc}$ ), 4.46 (s, 2H, H-Fc), 4.05 (s, 5H, $\mathrm{H}-\mathrm{Fc}$ ); IR (KBr) v: 3162, 3020, 2930, 1661, 1607, 1509, $1303,1187,1079,883,821,757,687 \mathrm{~cm}^{-1}$. Anal. calcd for $\mathrm{C}_{25} \mathrm{H}_{18} \mathrm{ClFeN}_{3} \mathrm{OS}$ : C 60.08, N 8.41, H 3.63, S 6.42; found C 60.01, N 8.39, H 3.70, S 6.37.

$N$-[5-(4-溴基苯基)-1,3,4-噻二唑-2-基]-4-二茂铁苯 甲酰胺 $\left(\mathrm{FcL}_{5}\right)$ : 展开剂: $V$ (二氯甲烷 $): V($ 甲醇 $)=20 ： 1$, 橙红色固体, 产率 $28 \%$. m.p. $214 \sim 216{ }^{\circ} \mathrm{C} ;{ }^{1} \mathrm{H}$ NMR (DMSO- $\left.d_{6}, 600 \mathrm{MHz}\right) \delta: 8.09$ (d, $J=8.5 \mathrm{~Hz}, 2 \mathrm{H}, \mathrm{H}-\mathrm{Ar}$ ), 7.87 (s, $J=8.1 \mathrm{~Hz}, 2 \mathrm{H}, \mathrm{H}-\mathrm{Ar}$ ), 7.69 (d, $J=8.5 \mathrm{~Hz}, 2 \mathrm{H}$, H-Ar), 7.62 (s, $J=8.1 \mathrm{~Hz}, 3 \mathrm{H}, \mathrm{H}-\mathrm{Ar}$ ), 4.89 (s, $J=1.8$ $\mathrm{Hz}, 2 \mathrm{H}, \mathrm{H}-\mathrm{Fc}$ ), 4.41 (s, 2H, H-Fc), 4.04 (s, 5H, H-Fc); IR (KBr) v: 3144, 3010, 2930, 1669, 1607, 1517, 1303, 1115, 1071, 981, 821, $687 \mathrm{~cm}^{-1}$. Anal. calcd for $\mathrm{C}_{25} \mathrm{H}_{18} \mathrm{BrFeN}_{3} \mathrm{OS}$ : C 55,17, N 7.72, H 3.33, S 5.89; found C 55.15, N 7.67, H 3.33, S 5.82.

$N$-[5-(4-硝基苯基)-1,3,4-噻二唑-2-基]-4-二茂铁苯 甲酰胺 $\left(\mathrm{FcL}_{6}\right)$ : 展开剂: $V$ (二氯甲烷 $): V$ (甲醇 $)=20: 1$, 土褐色固体, 产率 $20 \%$. m.p. $281 \sim 283{ }^{\circ} \mathrm{C} ;{ }^{1} \mathrm{H}$ NMR (DMSO- $\left.d_{6}, 600 \mathrm{MHz}\right) \delta: 13.30(\mathrm{~s}, 1 \mathrm{H}, \mathrm{H}-\mathrm{N}), 8.38$ (d, $J=$
$8.5 \mathrm{~Hz}, 2 \mathrm{H}, \mathrm{H}-\mathrm{Ar}$ ), 8.29 (s, $J=8.1 \mathrm{~Hz}, 2 \mathrm{H}, \mathrm{H}-\mathrm{Ar}$ ), 8.11 (d, $J=8.5 \mathrm{~Hz}, 2 \mathrm{H}, \mathrm{H}-\mathrm{Ar}$ ), 7.74 (s, $J=8.1 \mathrm{~Hz}, 3 \mathrm{H}, \mathrm{H}-\mathrm{Ar}$ ), 4.97 (s, $J=1.8 \mathrm{~Hz}, 2 \mathrm{H}, \mathrm{H}-\mathrm{Fc}$ ), 4.47 (s, 2H, H-Fc), 4.06 (s, 5H, H-Fc); IR (KBr) v: 3154, 3010, 2920, 1661, 1607, 1517, 1455, 1313, 1187, 1097, 847, 749, $699 \mathrm{~cm}^{-1}$. Anal. calcd for $\mathrm{C}_{25} \mathrm{H}_{18} \mathrm{FeN}_{4} \mathrm{O}_{3} \mathrm{~S}$ : C 58.84, N 10.98, H 3.56, S 6.28; found C 58.81, N 10.92, H 3.64, S 6.28.

$\mathrm{N}$-[5-(4-三氟甲基苯基)-1,3,4-噻二唑-2-基]-4-二茂 铁苯甲酰胺 $\left(\mathrm{FcL}_{7}\right)$ : 展开剂: $V$ (乙酸乙酯) $: V($ 石油醚 $)=$ $1: 1$, 砖红色固体, 产率 $25 \%$. m.p. 294 $295{ }^{\circ} \mathrm{C} ;{ }^{1} \mathrm{H}$ NMR (DMSO- $\left.d_{6}, 600 \mathrm{MHz}\right) \delta: 13.18(\mathrm{~s}, 1 \mathrm{H}, \mathrm{H}-\mathrm{N}), 8.23$ (d, $J=8.5 \mathrm{~Hz}, 2 \mathrm{H}, \mathrm{H}-\mathrm{Ar}$ ), 7.89 (s, $J=8.11 \mathrm{~Hz}, 2 \mathrm{H}, \mathrm{H}-\mathrm{Ar}$ ), 7.92 (d, $J=8.5 \mathrm{~Hz}, 2 \mathrm{H}, \mathrm{H}-\mathrm{Ar}), 7.73$ (s, $J=8.1 \mathrm{~Hz}, 3 \mathrm{H}$, H-Ar), 4.96 (s, $J=1.8 \mathrm{~Hz}, 2 \mathrm{H}, \mathrm{H}-\mathrm{Fc}$ ), 4.47 (s, 2H, H-Fc), 4.05 (s, 5H, H-Fc); IR (KBr) v: 3172, 3020, 2920, 1679, 1607, 1517, 1303, 1169, 1107, 1069, 991, 829, $695 \mathrm{~cm}^{-1}$. Anal. calcd for $\mathrm{C}_{26} \mathrm{H}_{18} \mathrm{~F}_{3} \mathrm{FeN}_{3} \mathrm{OS}$ : C 58.55, N 7.88, H 3.40, S 6.01; found C 58.51, N 7.86, H 3.44, S 6.09.

\section{2 .4 单晶结构测试}

称取 $0.05 \mathrm{~g}$ 的 $\mathrm{FcL}_{4}$ 的粉末状固体溶于 $5 \mathrm{~mL}$ 的 DMSO 溶剂中, 加热使其尽量溶解, 过滤, 将清液静置 数天, 瓶子底部有红色晶体析出. 室温下, 挑选尺寸适 中的晶体, 置于 Bruker SMART APEX IICCD X 射线单 晶衍射仪上, 通过石墨单色器以 $\mathrm{Mo}-\mathrm{K} \alpha$ 单色射线 $(\lambda=$ $0.767 \AA)$ 作为衍射光源, 按照 $\omega-2 \theta$ 的变速扫描下收集单 晶的衍射点数据. 晶体结构最后采用 SHELXS-97 程序 直接解出, 并通过傅立叶技术对所有非氢原子的坐标和 各向异性采用全矩阵最小二乘法进行精修.

\subsection{5 电化学性质测试}

室温下, 分别将 $\mathrm{FcL}_{1} \sim \mathrm{FcL}_{7}$ 溶于 $N, N$-二甲基甲酰 胺(DMF)中, 配成浓度为 $5 \times 10^{-4} \mathrm{~mol} / \mathrm{L}$ 的待测液, 用 $0.1 \mathrm{~mol} / \mathrm{L}$ 的四丁基高氯酸铵(TBAP)的 DMF 溶液作为支 持电解质. 以玻碳电极为工作电极, 铂丝电极为辅助电 极, 银/氯化银为参比电极的三电极体系, 实验前向待测 液中通氮气 $10 \sim 20 \mathrm{~min}$, 除去氧气对化合物循环伏安的 影响. 在 $-0.35 \sim 2.0 \mathrm{~V}$ 的扫描范围内, 测试目标化合物 的循环伏安行为.

\section{3 目标化合物的生物活性测试}

\subsection{1 抗菌活性测试}

称取洗净去皮的马铃薯 $200 \mathrm{~g}$, 切成小丁后加入 $1000 \mathrm{~mL}$ 蒸馏水. 煮沸持续 $20 \mathrm{~min}$, 过滤后向滤液中加 葡萄糖和琼脂各 $20 \mathrm{~g}$, 加热搅拌至全溶. 趁热分装至 $250 \mathrm{~mL}$ 的锥形瓶中, $121{ }^{\circ} \mathrm{C}$ 下湿热灭菌 $30 \mathrm{~min}$ 得马铃 薯葡萄糖琼脂培养基(PDA)培养基. 称取 $20 \mathrm{mg}$ 样品溶 
于二甲基亚砜(DMSO)中, 分别配制成 $3000 \mathrm{mg} / \mathrm{L}$ 的待 测药液, 用 $1 \mathrm{~mL}$ DMSO 配成空白对照溶液, 取 $1 \mathrm{~mL}$ 的 待测药液与 $14 \mathrm{~mL}$ 融化的 PDA 培养基混匀, 最终药液 浓度为 $200 \mathrm{mg} / \mathrm{L}$, 制成含药 PDA 平板. 将培养好的病原 菌菌饼移入含药培养血中, 于 $28{ }^{\circ} \mathrm{C}$ 恒温培养箱中培养. $72 \mathrm{~h}$ 以后将培养血取出, 观察菌落生长情况, 以 3 次测 试平行菌落样品生长直径的平均值计算抑制率. 抑制率 $(\%)=[($ 对照菌落直径一含药菌落直径 $) /($ 对照菌落直径 $-0.4)] \times 100 \%$. 化合物对玉米禾谷镰刀菌的 $\mathrm{EC}_{50}$ 值作 用测试方法同上, 取最终药剂浓度为 $200 、 100 、 50 、 25$ 、 $12.5 \mathrm{mg} / \mathrm{L}$ 五个浓度梯度.

\subsection{2 抗癌活性测试}

以 DMSO 为溶剂将化合物 $\mathrm{FcL}_{1} \sim \mathrm{FcL}_{7}$ 配制成 5000 $\mathrm{mg} / \mathrm{L}$ 的母液, 再分别用 ROMI-1640 型细胞完全培养基 稀释成 $100 、 50 、 25 、 12.5 、 6.25 \mathrm{mg} / \mathrm{L}$ 五个浓度梯度. 测 试前将人体肿瘤细胞放置在培养箱中于 $37{ }^{\circ} \mathrm{C}$ 恒温、 $5 \%$ $\mathrm{CO}_{2}$ 、饱和湿度的条件下培养, 取对数生长期的细胞进 行实验; 分别将人体肿瘤细胞接种于 96 孔板中, 每孔接 种 $100 \mu \mathrm{L}$ 浓度约为 $4 \times 10^{4} \sim 8 \times 10^{4}$ 的细胞悬浮液; 将 不同浓度梯度的待测物 $\mathrm{FcL}_{1} \sim \mathrm{FcL}_{7}$ 加入到 96 孔板中, 放置在培养箱中继续培养 $12 \mathrm{~h}$. 移取 $10 \mu \mathrm{L}$ 的 CCK- 8 加 入到 96 孔板中, 继续放回 $37{ }^{\circ} \mathrm{C}$ 恒温培养箱中于相同条 件下培养 $2 \mathrm{~h}$, 用全波长酶标仪于 $450 \mathrm{~nm}$ 的波长下测定 各孔的 OD 值, 每种样品的每个浓度重复做 3 次, 记录 数据. 通过酶标仪测定所得吸光度计算细胞平均抑制 率. 抑制率 $(\%)=[($ 对照一实验 $) /($ 对照一空白 $)] \times 100 \%$. 根据五个浓度的抑制率曲线, 用 SPSS16.0 软件出每个 化合物的 $\mathrm{IC}_{50}$ 值.

辅助材料(Supporting Information) 化合物 $\mathrm{FcL}_{4}$ 的主 要键长键角, 目标化合物 $\mathrm{FcL}_{1} \sim \mathrm{FcL}_{7}$ 的核磁氢谱谱 图. 这些材料可以免费从本刊网站(http://sioc-journal. $\mathrm{cn} /$ )上下载.

\section{References}

[1] El-Behairy, M. F.; Aboul-Enein, M. N; El-Azzouny, A. A. S.; Saleh, O. A.; Maklad, Y. A.; Aboutabl, M. E.; Maghraby, A. S.; Eur. J. Chem. 2014, 5, 488.

[2] Liu, T.-T.; Wan, Y.-C.; Fang, H. Chin. J. Org. Chem. 2016, 36, 417 (in Chinese). (刘婷婷, 万义超, 方浩, 有机化学, 2016, 36, 417.)

[3] Yadagiri, B.; Gurrala, S.; Bantu, R.; Nagarapu, L.; Polepalli, S.; Srujana, G.; Jain, N. Bioorg. Med. Chem. Lett. 2015, 25, 2220.
[4] Yang, H.-W.; Guan, J.-J.; Gao, F.-X.; He, X.-P.; Wang, A.; Sun, D.-B.; Zhang, X.-Q.; Zhang, B.; Feng, Y.-Q. Chin. J. Org. Chem. 2015, 35, 129 (in Chinese).

(杨贺玮, 官俊杰, 高峰贤, 何欣平, 王安, 孙宝德, 张学强, 张 宝, 冯亚青, 有机化学, 2015, 35, 129.)

[5] Joseph, A.; Shah, C. S.; Kumar, S. S.; Alex, A. T.; Maliyakkal, N.; Moorkoth, S. Acta Pharm. 2013, 63, 397.

[6] Megally Abdo, N. Y.; Kamel, M. M. Chem. Pharm. Bull. 2015, 63, 369.

[7] Suzuki, K.; Hamada, Y.; Nguyen, J. T.; Kiso, Y. Bioorg. Med. Chem. 2013, 21, 6665.

[8] Dekhane, D. V.; Pawar, S. S.; Gupta, S.; Shingare, M. S.; Patil, C. R.; Thore, S. N. Bioorg. Med. Chem. Lett. 2011, 21, 6527.

[9] Harish, K. P.; Mohana, K. N.; Mallesha, L.; Russ. J. Bioorg. Chem. 2014, 40, 97.

[10] Hu, Y.; Li, C.-Y.; Wang, X.-M.; Yang, Y.-H.; Zhu, H.-L. Chem. Rev. 2014, 114, 5572.

[11] Luo, Z.-H.; Chen, B.-Q.; He, S.-Y.; Shi, Y.-P.; Liu, Y.-M., Li, C.-W. Bioorg. Med. Chem. Lett. 2012, 22, 3191.

[12] Wu, K.-L.; Zhang, W.-B.; Zhou, D.; Xu, Y. Chin. J. Org. Chem. 2014, 34, 1201 (in Chinese). (吴孔丽, 张吾斌, 周丹, 徐琰, 有机化学, 2014, 1201.)

[13] Liu, Y.-T.; Wang, J.; Yin, D.-W. Fine Chem. 2013, 30 (in Chinese). (刘玉婷，王捷，尹大伟，精细化工, 2013, 30.)

[14] Zhang, Q.; Zhao, B.; Song, Y.; Hua, C.; Gou, X.; Chen, B. Heteroat. Chem. 2015, 26, 348.

[15] Plażuk, D.; Zakrzewski, J.; Salmain, M.; Błauż, A.; Rychlik, B.; Strzelczyk, P. Organometallics 2013, 32, 5774.

[16] Chopra, R.; De Kock, C.; Smith, P.; Chibale, K.; Singh, K. Eur. J. Med. Chem. 2015, 100, 1.

[17] Caballero, A.; Lloveras, V.; Curiel, D.; Tárraga, A.; Espinosa, A.; García, R.; Veciana, J. Inorg. Chem. 2007, 46, 825.

[18] Gou, X.-F.; Li, Y.; Chen, B.; Gao, X.-Y. Chem. Reag. 2013, 35, 305 (in Chinese). (苟小锋, 李媛, 陈邦, 高学祥, 化学试剂, 2013, 35, 305.)

[19] Yin, D.-W.; Sun, X.-M; Liu, Y.-T. Appl. Mech. Mater. 2012, 189, 181.

[20] Quintana, C.; Klahn, A. H.; Artigas, V.; Fuentealba, M.; Biot, C.; Halloum, I.; Halloum, I.; Kremer, L.; Arancibia, R. Inorg. Chem. Commun. 2015, 55, 48.

[21] Gu, L.-Q.; Li, J.; Fu, Y.-L.; Ran, C.-L.; Xu, Y.; Fan, Y.-T. Chin. J. Inorg. Chem. 2010, 23, 2113 (in Chinese).

(顾立强, 李杰, 傅亚丽, 再春玲, 徐琰, 樊耀亭, 无机化学学报, 2010, 26, 2113.)

[22] Feng, Z.-J.; Wu, D.-L.; Shang, Y.-J. Chin. J. Synth. Chem. 2007, 15, 154 (in Chinese). (冯志君, 吴德林, 商永嘉, 合成化学, 2007, 15, 154.)

[23] Xu, Y.; Zhu, L.-M.; Ran, C.-L.; Wang, H.-X.; Fan, Y.-T. Chin. J. Inorg. Chem. 2007, 23, 589 (in Chinese). (徐琰, 朱丽敏, 再春玲, 王海先, 樊耀亭, 无机化学学报, 2007, 23, 589.)

[24] Khan, N.; Badshah, A.; Lal, B.; Malik, M. A.; Raftery, J.; O'Brien, P.; Altaf, A. A. Polyhedron 2014, 69, 40.

[25] Nikalje, A. P. G.; Shaikh, S. I.; Khan, F. A. K.; Shaikh, S.; Sangshetti, J. N. Med. Chem. Res. 2015, 24, 4058. 